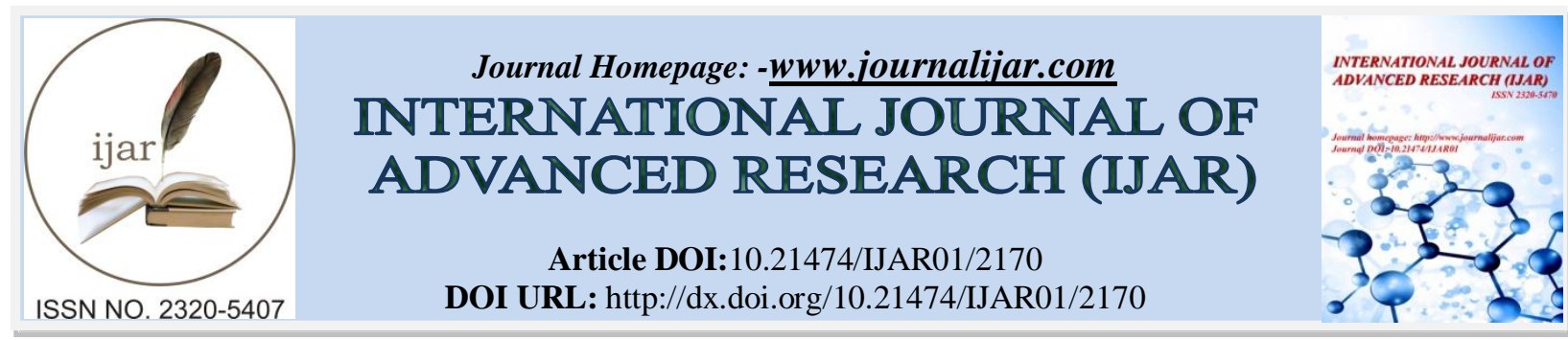

RESEARCH ARTICLE

\title{
A CASE STUDY ON EFFICACY OF TRANSCRANIAL DIRECT CURRENT STIMULATION IN MANAGEMENT OF MIGRAINE.
}

Manoj Malik ${ }^{1}$, Shailendra Kumar Singh ${ }^{2}$ and Narkeesh Arumugam ${ }^{3}$.

1. Assistant Professor, Department of Physiotherapy, Guru Jambheshwar University of Science and Technology, Hisar.

2. Professor, Department of Pharmaceutical Sciences, Guru Jambheshwar University of Science and Technology, Hisar.

3. Professor, Department of Physiotherapy, PunjabiUniversity, Patiala.

\section{Manuscript Info}

Manuscript History

Received: 26 September 2016

Final Accepted: 29 October 2016

Published: November 2016

Key words:-

TDCS, Migraine, TES, Headache

\section{Abstract}

Objective- To describe the effect of Transcranial direct current stimulation (TDCS) in Management of Migraine.

Background- Patient was a forty years old male with complain of migraine headaches since 2011.Patient described them as tight unilateral band present over temple with moderate to severe aversion to light and sound during attack.

Method- Patient was treated thrice a week for six weeks.TDCS was applied at an intensity of 2 ma for 20 minutes. Cathode was placed at $\mathrm{Cz}$ and anode over $\mathrm{Oz}$ according to 10-20 EEG placement.

Result- Patient reported a decrease in headache disability index, Headache impact test 6 (HIT6) scores as well as headache duration, frequency and intensity. Conclusion-TDCS can be an effective technique in management of migraine.

Abbreviations- TDCS- Transcranial Direct Current Stimulation, TMSTranscranial magnetic Stimulation, TES-Transcranial Electrical Stimulation.

Copy Right, IJAR, 2016,. All rights reserved.

\section{Introduction:-}

Migraine is a central nervous system disorder characterized by severe headaches and other associated symptoms. Nausea occurs in almost $90 \%$ of patients while vomiting occurs in about $1 / 3^{\text {rd }}$ of patients. Many patients experience sensory hyper excitability manifested by photophobia, phonophobia etc ${ }^{1}$.According to World Health Organization, Migraine affects about $15 \%$ of population. It is most common neurological disease and is ranked $12^{\text {th }}$ among women and $19^{\text {th }}$ in general population for the degree of handicap it causes ${ }^{2}$.Prevalence of Migraine headache is higher in women $(18 \%)$ than in men $(6 \%)^{3}$. There may be localized edema of scalp or face or scalp tenderness, prominence of vein or artery in the temple or stiffness and tenderness of the neck ${ }^{1}$.Migraine is a neurovascular disorder and symptoms arise from combination of vascular and neurological events occurring in the cranial meninges.Cortical spreading depression, an electrophysiological event in Migraine, is described as an intense wave that propagates across the cerebral cortex at a rate of $2-5 \mathrm{~mm} /$ minute lasting for 15 to 30 minutes causing disruption of ionic gradients followed by a period of suppressed neural activity ${ }^{4}$.These mechanisms include the release of inflammatory cytokinens,neuroinflammatory peptides and calcitonin gene related peptide. Activation of trigeminovascular system during pain phase of Migraine is thought to initiate a cascade of chemical activity from trigeminal sensory nerve 
endings. Subsequent to initiation of migraine by either CSD or brainstem generator, meningeal pain mechanism commence through trigemino-vascular activation ${ }^{3}$. Migraine prophylaxis requires daily administration of anti migraine compounds whether or not migraine attack is occurring. All the drugs used for migraine prevention have potential and often relevant adverse effects or contraindications, and may also interfere with other concurrent conditions and treatment . These problems may induce patient to reject the idea of a preventive treatment or may lead to poor patient adherence ${ }^{5}$.Adverse effects of prophylactic medications, contraindications due to existing conditions like Asthma, Myocardial Infarction etc ,are primary reasons of patient avoiding medications and poor patient compliance. A number of alternate treatment methods like Manual therapy, Neuromodulatory techniques, biofeedback, dietary changes and behavioral therapy approaches have been extensively studied for their efficacy in prophylaxis of Migraine.

Application of electrical current to modify brain functions is a very old technique, mentioned more than 200 years ago. Non Invasive Brain stimulation techniques are emerging as promising methods of altering the plasticity response. Inparticular, transcranial direct current stimulation is a new technique with potential to induce change in cortical networks which outlast the period of stimulation .TDCS is a non-invasive brain stimulation technique that applies a mild (1 to $2 \mathrm{ma}$ ) direct electrical current via the scalp to enhance or diminish neuronal excitability. There is strong evidence that neurons underlying the anode are excited with resting membrane potential shifting towards depolarization and an increased rate of spontaneous neuronal firing and the neurons underlying the cathode are inhibited with resting membrane potential shifting towards hyper polarization and reduced neuronal firing ${ }^{6}$.

Generation of electrochemically produced toxins and electrode dissolution products at the electrode tissue interface are the only risks of TDCS for the skin contact, because there is no brain electrode interface ${ }^{7}$ TDCS is a portable,safe,non-invasive brain stimulation technique that is capable of modulating the excitability of targeted brain regions by altering neuronal membrane potentials based on polarity of the current transmitted through the scalp via sponge electrodes ${ }^{8}$. TDCS as a neuromodulatory technique can be safe and effective in Management of Migraine.

\section{Case Study:-}

A 40 year old male presented in April 2016 with headaches that began in 2011.There was no precipitating factor; however patient was in stress due to some family issues. Patient had unilateral headaches lasting for four to six hours with moderate to severe pain intensity. Patient described headaches attacks as tight unilateral band present over temple with moderate to severe aversion to light (photophobia) and sound (Phonophobia) during the attack. Patient reported history of irritable bowel syndrome and vague muscle aches.He was not taking any prophylactic medication for migraine .Patient did not apply any herbal hair oil massage or any other type of treatment for his migraine headaches. Patient was asked to fill headache disability index and headache impact test 6 Questionnaire. He was given headache diary to report duration, frequency and intensity of his headaches. Transcranial direct current stimulation was applied to patient for six weeks, thrice a week on alternate days except Sunday. Cathode was placed over $\mathrm{Cz}$ and anode over $\mathrm{Oz}$ according to 10-20 EEG Placement system. Intensity was kept at 2 ma for 20 minutes during each visit. Patient was asked to report any adverse effect like neck pain, scalppain, tingling, itching, rednessetc after every session. Patient did not report any adverse event during the treatment .Patient was asked to fill headache diary during the period of treatment .Patient reported significant reduction in headache frequency, duration and frequency by the end of treatment as well as in scores of headache disability index and headache impact test.

\begin{tabular}{|l|l|l|}
\hline Scale & Pre Intervention Scores & Post Intervention Scores \\
\hline Headache Disability Index & 66 & 32 \\
\hline Headache Impact Test 6 & 67 & 55 \\
\hline Headache Frequency/per month & 5 & 3 \\
\hline Headache Intensity & 9.2 & 6.33 \\
\hline Headache Duration & 4 hours 12 min & $2 \mathrm{hrs} 20 \mathrm{~min}$ \\
\hline
\end{tabular}




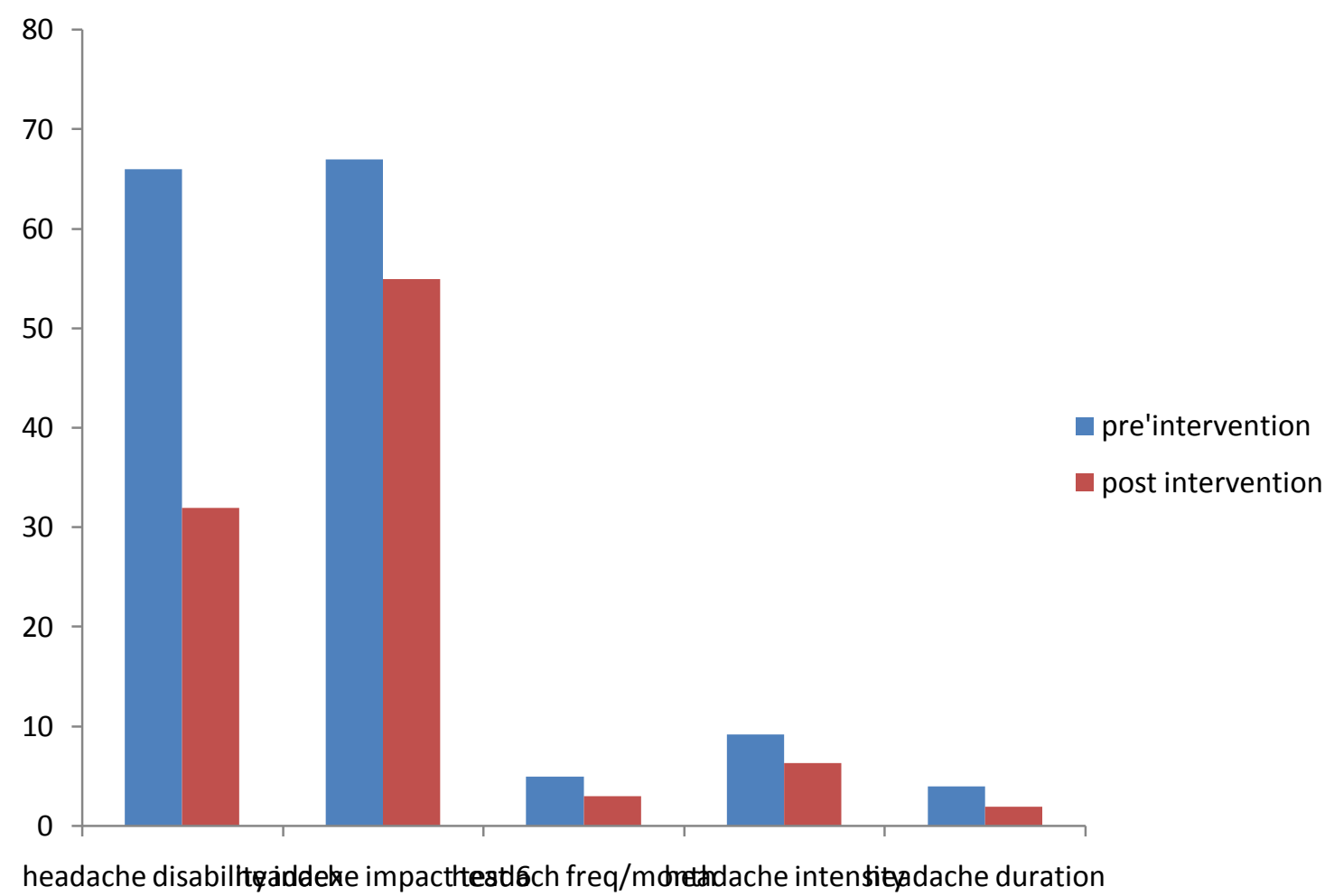

Fig 1:- pre and post intervention data for HDI, HIT-6, and Headache Diary

\section{Discussion:-}

Headache is a common disease, its more severe manifestations such as intractable migraine and trigeminal autonomic cephalgia have a debilitating effect on patients resulting in pain and severe functional impairment.TDCS applied through the skull has been shown to directly modulate the excitability of cortical areas.Cathodal stimulation induces a decrease and anodal stimulation an increase of cortical excitability. Pharmacological studies show that effects during stimulation are mediated by ion channels in accordance with primary hyper or depolarizing effect of stimulation while after effects involve the modulation of N-Methy D Aspartate(NMDA) Receptor efficacy. Based on the concept of cortical hyper excitability in migraine, cathodal TDCS in migraine is expected to normalize the cortical excitability either by prophylactic treatment in interictal phase or by an acute treatment in beginning of migraine $\operatorname{attack}^{10}$.

Gate Control theory given by wall and melzack provided a foundation for considering direct electrical stimulation of spinal cord and peripheral nerves as apossible treatment for Chronic pain ${ }^{11}$.Manjit S Matharee et al described eight cases of chronic migraine who had responded very well to implanted bilateral sub occipitalstimulation. Patients reported that stimulation rapidly suppressed the pain and it recurs instantaneouslywhen stimulation is ceased .Patient were able to either completely discontinue or markedly decrease the intake of abortive and preventive medications for headaches ${ }^{12}$.

It is likely that migraine episodes are caused by events that should be described as transitions in the dynamic state of brain and it can take various triggers to make the transition but the cause is inherently in the dynamics. Since, dynamical disease are caused by transitions in both temporal rhythms and spatiotemporal patterns, neuromodulation is natural ansatz for their treatment .Transcranial stimulation targets the cortex. Two non invasiveneuromodulation techniques are available in Migraine, TMS and TES. Preliminary evidence was found for patients with migraine having a positive but delayed response to TDCS applied to motor (anodal) and Orbitofemoral (Cathode) Cortices ${ }^{13}$. 
TDCS has been shown to be effective in Management of Migraine. Alexandra F dasliva et al performed a Randomized controlled trial with ten sessions of over four week duration of Anodal TDCS. Result showed positive but delayed response to anodal TDCS of primary motor cortex.PardeeAuvichayapat et al performed a Randomized placebo controlled trial to evaluate the efficacy of Anodal TDCS in Migraine Prophylaxis .They concluded that Anodal MI TDCS may be safe and useful clinical tool in Migraine prophylaxis ${ }^{3}$.In the current case improvements are seen in headache frequency, duration and intensity as well as in scores of HIT 6 and headache disability index.

Migraine is a chronic disease related with disability and reduced quality of life.Prophylatic pharmacological management has serious side effects and poor compliance. Various studies have been performed to evaluate the efficacy of Physical and behavioral treatment approaches in Management of Migraine. These treatments may have better acceptability in Migraine patients due to fewer side effects. In the present case, no serious adverse effect was noted by patient during or after application of TDCS.

\section{Conclusion:-}

Present case study provided a detailed account of effects of transcranial direct current stimulation on headache duration, frequency, intensity and headache related disability in a patient suffering from Chronic Migraine. This case study may indicate the efficacy of TDCS in management of migraine. However, This is a case study of one patient and results cannot be generalized for patients suffering from Chronic Migraine.

\section{References:-}

1. SalomoneS, CaraciF, CapassoA.Migraine: AnOverview.The open Neurology Journal.2009; 3:64-71.

2. SprengerT, Borsook D.Migraine changes the Brain-Neuroimaging Imaging makes its mark. Current Opinion Neurology.2012; 25(3):252-262.

3. Auvichayapat P, Janyachareon T, Rotenberg A, Tiamkao S, Krisanaprakronkit T, Sinawat S, PunjarukW, Thinkhamrop B, Auvichayapat N. Migraine Prophylaxis by Anodal TranscranialDirectCurrentStimulation, a Randomized ,Placebo-Controlled Trial.Journal of the Medical Association of Thailand.2012;95(8):1003-1012.

4. Viganò A, D'Elia TS, Sava SL, Auvé M,Pasqua VD, Colosimo A, Piero VD, Schoenen J, MagisD.Transcranial Direct current Stimulation of the Visual cortex ; a proof of concept studybased on interictal electrophysiological abnormalties in Migraine.The Journal ofheadache and Pain.2013;14(1):23. doi: 10.1186/1129-2377-14-23.

5. D'Amico D, TepperSJ.Prophylaxis of Migraine: general principle and patient acceptance. Neuropsychiatric disease and treatment .2008; 4(6):1155-1167.

6. SchabrunSM,Transcranial direct current stimulation: a place in the future of physiotherapy. Physical therapy reviews.2010; 15(4):320-326.

7. NitscheAM,CohenLG,WassermanME,PrioriA,LangN,AntalA,PaulasW,HummelF,BoggioPS,PascaulleoneA.Transcranial direct current stimulation:state of art 2008.Brain Stimulation.2008;1:206-223.

8. Schlaung G, RengaV.Transcranialdirect current stimulation: a non-invasive tool to facilitate stroke recovery. Expert Review Medical Devices.2008:5(6); 759-768.

9. MartelletiP,JensenRH,AntalA,ArcioniR,BrighinaF,TomassoMD,FranziniA,FontaineD,HeilandM,JurgensTP,Le oneM,MagisD,PaemeleireK,PalmisaniS,PaulasW,May A. Neuromodulation of chronic headaches: Position statement from the European headache federation. The Journal of headache and Pain.2013; 14:1-16.

10. Reed KL, Peripheral neuromodulation and headaches: History, clinical approach and considerations in underlying mechanism.Current Pain and Headache reports.2013; 17:305DOI 10.1007/s11916-012-0305-8.

11. MatharuMS,BartschT,WardN,RichardSJ,WeinerR,GoadsbyPJ.Centralneuromodulation in chronic migraine patients with sub occipital stimulators: a PET Study.Brain.2004;127:220-230.

12. Dahlem MA, Migraine generator network and spreading depression dynamics as neuromodulation targets in episodic migraine, Chaos, 2013:23:046101-12.

13. DasilvaAF,MendoncaME,ZaghiS,LopesM,DossantosMF,Spierings EL, BajwaZ,DattaA,Bikson M, FregniF.TDCS Induced Analgesia and Electrical fields in pain related neural networks in Migraine.Headache.2012;52(8):1283-1295. 